\title{
RAYONNEMENT ACOUSTIQUE DES COQUES CYLINDRIQUES, MUNIES D'UN MATERIAU DE MASQUAGE
}

\section{B. LAULAGNET et J.L. GUYADER}

Laboratoire Vibrations-Acoustique, Bât. 303, INSA de Lyon, F-69621 Villeurbanne Cedex, France

RESUME : Cet article présente la modèlisation du rayonnement acoustique d'une coque cylindrique revêtue d’un matériau de masquage. Deux approches différentes sont proposées ; dans la première le matériau est modèlisé par une raideur complexe, à réaction localisée, interposée entre le fluide extérieur et la coque. Cette description du matériau de masquage, bien quapproximative, rend compte du phénomène de découplage entre le fluide et la coque, à partïr d'une fréquence de coupure. Cependant, le lien entre la raideur localisée et les caractéristiques du matériau (Module de Young, coefficient de PoIsson) n'est pas facile à faire. On peut aussi affirmer que l'hypothèse de réaction localisée n'est pas adaptée aux matériaux incompressibles, aussi proposons nous un second modèle basé sur la résolution approchée des équations de Navier du matériau, par développement asymptotique dans l'épaisseur de la couche. L'analyse des résultats obtenus permet de comparer les deux modèlisations et met en évidence les mécanismes de rayonnement et d'isolement de la coque munie d'une couche de matériau de masquage.

ABSTRACT : An analytical study of radiation by finite, cylindrical shell covered with a compliant layer is presented using two different approaches. Firstly, the compliant layer is described by a locally reacting model, as a radial spring acting between the fluid loading and the shell displacements. This method leads to the clarification of the shell's vibro-gcoustic behavior, but infortunately cannot take inta account, mains caracteristics of the layer such as, compressibility. The second model can do this and consists in solving Navier's equations using asumptotic expansions in the thickness lauer, supposed sufficiently "thin". Results are presented in shell radial quadratic velocity and acoustic radiated power, which point out the mecanisms of radiation/isolation encountered when the shell is covered.

\section{INTRODUCTION}

Lors de précédentes études, nous avons proposé une méthode de calcul du rayonnement acoustique des coques culindriques, finies, munies de raidisseurs [1], [2], [3]. Ces travaux font clairement apparaitre la difficulté de diminuer le bruit émis sans modification importante de la structure; par contre la couverture dune coque par une couche de matériau viscoélastique qui permet de réduire la puissance acoustique rayonnée, de façon considérable [4], [5], apparaît comme une solution aux problèmes de discrètion acoustique des coques immergées. L'objet de cette publication est de proposer deux modèlisations théoriques du rayonnement des coques reyêtues de matériau de masquage prenant en compte le caractère modal de la réponse de la coque.

Dans la première approche, le matériau est vu comme une raideur locale, complexe, à l'interface de la coque et du fluide extérieur. Cette approche qui présente l'ayantage d'être simple, rend compte du phénomène principal. Le deuxième modèle décrit le matériau de façon plus réaliste et fait appel aux equations de l'élasticité tridimensionnelle. Nous sommes alors à même de chiffrer l'influence des grandeurs caractéristiques d'un problème de masquage, notamment le facteur de compressibilité de la couche, qui gouverne l'isolation. Les résultats numériques présentés feront lobjet de la troisième partie de cet 
article ou les deux modèles seront comparés.

\section{MODELISATION DU MATERIAU DE MASQUAGE A REACTION LOCALISEE}

2.1. Modèle du matériau à réaction localisée

L'idée de base de cette modèlisation est de considèrer la couche de matériau yisco-élastique comme un ressort sans masse et sans épaisseur. On pase, pour la pression acoustique pariétale au point $Q$ :

$$
p(0)=z\left(w_{a}-w_{c}\right)
$$

où $Z$ est l'impédance localisée complexe de la couche visco-élastique, homogène à une raideur par unité de surface $; w_{a}$ et $w_{c}$ sont respectivement les déplacements acoustiques de la face externe de la couche (en contact avec lé milieu fluide) et de la caque. Dans la mesure où l'impédance est complexe, nous pouvons établir le schéma suivant:

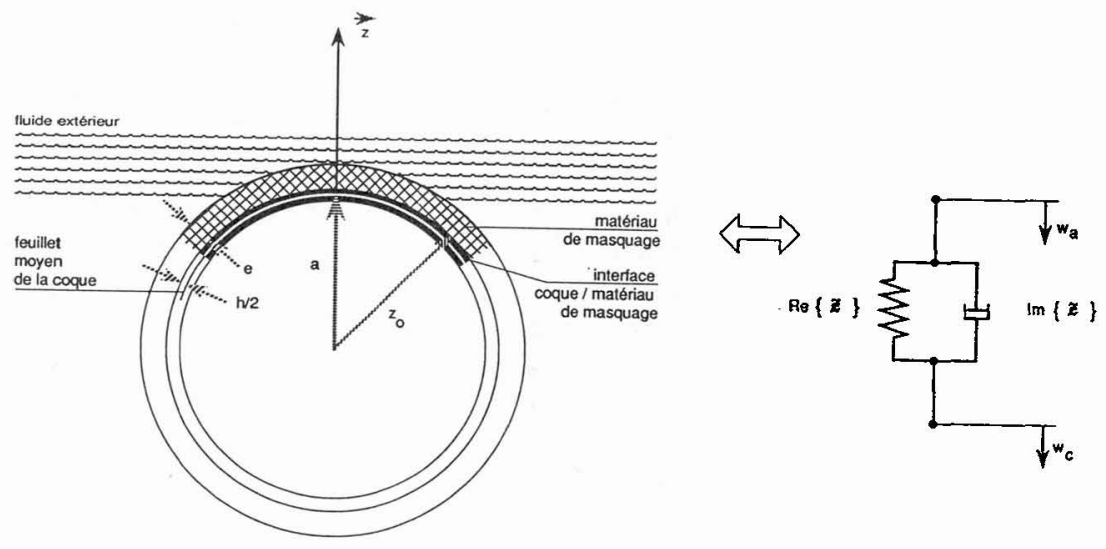

Figure 1 : Schema synoptique de la coque recouverte d'une couche de materiau de masquage et système de coordonées

2.2. Expression de la pression pariétale pour une coque recouverte de matériau de masquage

La coque est immergée dans un fluide de volume $W$ infini. L'acoustique interne est négligée. Les équations qui régissent le couplage fluide structure en régime harmonique $\left(e^{-j \omega t)}\right.$ sont les suivantes:

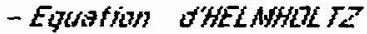

$$
\Delta p(M)+w^{2} / c^{2} p(M)=0 \quad M \in V \quad \text { (2) }
$$

avec les conditions de SOMMERFELD pour des rauons infinis. $p(M)$ est la pression acoustique et $c$ la célérité des ondes dans $\psi$. Nous n'avons plus la continuité des vitesses mécarique et acoustique à l'interface coque-fluide puisque nous avons interposé le matériau de masquage entre les deux. Au contraire, nous avons une discontinuité de vitesse, sur la coque, décrite par la relation (3.a) et sur le baffle une vitesse nulle : 


$$
\begin{array}{lll}
p(Q)=Z\left[\left(1 /\left(\rho_{0} \omega^{2}\right) \partial p / \partial z(Q)-w(Q)\right]\right. & Q \in s & (3 . a) \\
(\partial p / \partial z)(Q)=0 & Q \in s_{b} & (3 . b)
\end{array}
$$

$1 /\left(\rho_{0} \omega^{2}\right) \partial p / \partial z(Q)$ est en fait le déplacement acoustique en "face externe" du visco-élastique ou $\hat{P}_{0}$ est la masse volumique de fluide exterieur. On remarque immédiatement que lorsque $Z$ prend des valeurs infiniment grandes, le rapport $p(0) / Z$ tend vers 0 . On obtient alors l'égalité entre le déplacement acoustique et le déplacement mécanique de la coque au point Q. On retombe alors sur le problème exposé en [1], [2].

Pour déterminer la pression pariétale au point $Q$, nous adjoignons le problème de GREEN suivant:

$$
\begin{array}{ll}
\Delta G\left(M, Q_{0}\right)+w^{2} / c^{2} G\left(M, Q_{0}\right)=0 & M \in V \\
\left.\partial G / \partial z\left(Q, Q_{0}\right)=-\delta Q D-Q_{0}\right) & \left(Q_{0}, D\right) \in S
\end{array}
$$

avec les conditions de SOMMERFEL pour $z$ infini. $\delta$ est la distribution de Dirac. Appliquans le théorème de GREEN au volume extérieur au cylindre bafflé et utilisons les conditions de vitesse $3 a$ et $3 b$, il vient :

$$
\int_{S} \partial G / d z\left(Q, Q_{0}\right) p(Q) d S=\rho_{0} \omega^{2} \int_{S}(w(Q)+\rho(Q) / Z) G\left(0, Q_{0}\right) d S
$$

En appliquant la condition (5) et en permutant les point $Q$ et $Q_{0}$, on a la pression pariétale au point $Q$ qui prend la forme suivante:

$$
p(Q)=-\rho_{0} \omega^{2} \int_{S}\left(w\left(Q_{0}\right)+p\left(Q_{0}\right) / 2\right) G\left(Q_{,} Q_{0}\right) d S
$$

L'expression (7) exprimée à partir d'un potentiel de simple couche, donne la pression pariétale en tout point de la surface 5 . La présence du matériau de masquage d'impédance $Z$, fait qu'elle dépend non seulement du déplacement radial de la coque mais aussi, explicitement de la pression.

2.3. Equation du mouvement de la coque immergée, revêtue du matériau de masquage, décomposée dans la base modale de la coque appuyée sur ses bords.

L'équation du mouvement de la coque sous charge fluide, soumise à une excitation ponctuelle appliquée en $\mathrm{M}_{e}$ est la suivante:

$$
\left.E h /\left(1-v^{2}\right)[L]\left\{\begin{array}{l}
u \\
v \\
v
\end{array}\right\}+\rho h \omega^{2}\left\{\begin{array}{l}
u \\
v \\
v
\end{array}\right)=-\left\{\begin{array}{l}
F_{X} \\
F_{\Psi} \\
F_{z}
\end{array}\right\} \delta\left(M-M_{e}\right)+\mid \begin{array}{c}
0 \\
0 \\
p(Q)
\end{array}\right\}
$$

où $(u, v, w)$ sont les composantes du déplacement de $Q$ en coordonnées cylindriques $(\vec{x}, \vec{v}, \vec{z})$, $\left(F_{X}, F_{\psi}, F_{Z}\right)$ les composantes de la force, [L] est l'opérateur de FLUGGE, $E$ le modèle d'YOUNG de la coque, son coefficient de POISSON, $h$ son épaisseur, $\rho$ sa masse volumique.

La résolution s'effectue par décomposition des fonctions dansla base des modes propres de la coque in yacuo, appuyée sur ses bords (d'autres cas de conditions aux limites peuyent être envisagés mais n'entrent pas ici dans notre propos). Les déformées modales de la 
coque sont de la forme:

$$
\vec{\Phi}_{n m j}^{\alpha}(x, \phi)=\left\{\begin{array}{c}
D_{n m j} \sin (n \psi+\alpha \pi / 2) \cos m \pi x / L \\
E_{n m j} \cos (n \psi+\alpha \pi / 2) \sin m \pi x / L \\
1 \sin (n \varphi+\alpha \pi / 2) \sin m \pi x / L
\end{array}\right\}
$$

où $L$ est la longueur de coque entre appuis, $\alpha$ le type de mode $(0$, antisymétrique ; 1 symètrique), $n$ lordre circonférentiel, $m$ lordre axial, j le tupe du mode (à prépondérance de flexion, torsion, traction-compression), $D_{n m j}, E_{n m j}$, 1) les composantes du déplacement modal. On écrit alors pour la pression pariétale:

$$
\left\{\begin{array}{l}
0 \\
0 \\
p(0)
\end{array}\right\}=\sum_{\alpha=0}^{1} \sum_{n=0}^{\infty} \sum_{m=1}^{\infty} \sum_{j=1}^{3} b^{\alpha}{ }_{n m j} \quad \mathbf{\Phi}_{n m j}^{\alpha}(0)
$$

et pour des déplacements de la coque:

$$
\left\{\begin{array}{l}
u \\
v \\
v
\end{array}\right\}=\sum_{\alpha=0}^{1} \sum_{n=0}^{\infty} \sum_{m=1}^{\infty} \sum_{j=1}^{3} a_{n m j}^{\alpha} \quad \vec{\Phi}_{n m j}^{\alpha}
$$

Avec les expressions $(10)$ et $(11)$, on passe des inconnues $(u, v, w, P)$ aux inconnues modales du déplacement de la coque $a^{\alpha}{ }_{n m j}$, et de la pression pariétale $b_{n m j}^{\alpha}$. La projection des équations (7) et (8) dans la base des modes, après utilisation des orthogonalités dorne tous calculs faits pour (7):

$$
b_{p q k}^{\alpha} N_{p q k}=-j \omega \sum_{m=1}^{\infty} \sum_{j=1}^{3} z_{p q m}\left(a_{p m j}^{\alpha}+b_{p m j}^{\alpha} / Z\right)
$$

pour (8):

$$
n_{p q k}\left(\omega_{p q k}^{2}\left(1-m_{c}\right)-\omega^{2}\right) a_{p q k}^{\alpha}=F_{p q k}^{\alpha}-b_{p q k}^{\alpha} N_{p q k}(13)
$$

$\eta_{c}$ est le facteur de pertes structurales de coque, $N_{p q k}$, la norme du mode de coque, $m_{\rho q k}$ sa masse généralisée, $F_{p q k}^{\alpha}$ la force généralisée et $Z_{p q m}$ est l'impédance de rayonnement, quantité complexe qui rend compte du couplage fluide-structure, mais aussi du couplage intermodal par rayonnement. Son expression est la suiyante (cf. [1] [2]) :

$$
z_{p q m}=j \rho_{0} w a^{2} / \varepsilon_{p} \int_{-\infty}^{+\infty} H_{p}\left(k_{z} \text { a) } \tilde{\Psi}_{m}\left(k_{x}\right) \tilde{\Psi}_{q}^{*}\left(k_{x}\right) /\left[k_{z} \text { a } H_{p}\left(k_{z} a\right)\right] d k_{x} \quad\right. \text { (14) }
$$

avec $H_{p}$ et $H_{p}$ fanctions et dérivées de fonction de HANKEL d'ordre $p$, de première espèce, garantissant des ondes se propageant vers les rayons croissants; a rayon de la coque, $\mathrm{c}_{\mathrm{p}}$ facteur de Neuman, $k$, nombre d'onde acoustique, $k_{x}$ composante axiale du nombre d'onde tel que $k_{x}=\left(k^{2}-k_{z}\right)^{1 / 2}, \tilde{\Psi}_{m}\left(k_{x}\right)$ et $\tilde{\psi}_{q}^{*}\left(k_{x}\right)$ sont les transformées de Fourier spatiales de formes propres axiales données par: 


$$
\begin{aligned}
& \tilde{\psi}_{m}\left(k_{x}\right)=\int_{0}^{L} \sin (m \pi / L) x e^{-i k_{x} x} d x \\
& \tilde{\Psi}_{q}\left(k_{x}\right)=\int_{0}^{L} \sin (q \pi / L) x e^{i k_{x} x} d x
\end{aligned}
$$

Substituons (13) dans (12), on obtient finalement le système linéaire suivant:

$$
\begin{aligned}
& m_{p q k}\left(\omega_{p q k}^{2}\left(1-j n_{c}\right)-w^{2}\right) a^{\alpha}{ }_{p q k} \\
& -j \omega \sum_{m=1}^{\infty} \sum_{j=1}^{3} z_{p q m}{ }^{\alpha}{ }_{p m j}\left[1-m_{p m j}\left(\omega^{2}{ }_{p m j}\left(1-j \eta_{c}\right)-\omega^{2}\right) /\left(Z N_{p m j}\right)\right] \\
& =F_{p q k}^{\alpha}+j \omega \sum_{m=1 j=1}^{\infty} \sum_{p q m}^{3} F_{p m j}^{\alpha} /\left(Z N_{p m j}{ }^{\alpha}\right.
\end{aligned}
$$

La résolution des systèmes linéaires (16) permet le calcul des grandeurs physiques intéressantes tel que puissance acoustique rayonnée et vitesse quadratique radiale de coque.

\section{MODELISATION DU MATERIAU OE MASQUAGE PAR L'ELASTICITE TRIDIMENSIONHELLE}

Nous présentons dans cette partie, la modèlisation du matériau de masquage au moyen de l'élasticité tridimensionnelle. Les déplacernents dans la couche visco-élastique sont régis par un système linéaire d'ordre 3, d'équations différentielles du second ordre. En supposant que la couche est suffisamment "mince", des solutions sont recherchées sous forme de développements asymptotiques dans l'épaisseur du matériau. La méthode consiste à écrire la continuité des déplacements de la coque et du matériau à l'interface, ainsi qu'à raccorder la contrainte normale avec la pression acoustique pariétale, à l'interface du matériau et du fluide extérieur. Ceci aboutît à la construction d'un système linéaire, traduisant le couplage coque mince - matériau de masquage - fluide extérieur, exprimé à partir des déplacements de la coque et d'inconnues introduites par les développements asymptotiques, et dont le nombre dépend du degrế de précision exigé sur les solutions (et donc de l'ordre de la troncature des développements).

Nous traitons dans cet article du cas particulier ou les déplacements sont approchés par des développements asymptotiques tronqués au deuxième ordre, ce qui impose des contraintes décrites au premier ordre et des équations du mouyement yérifiées à l'ordre 0.

\subsection{Equations du mouvernent de la couche visco-élastique}

Le mouvement de la couche visco-élastique, déformable dans son épaisseur, est décrit par les équations de l'élasticité tridimensionnelle, en coordonnèes culindriques,cf [6]. Dans le cas d'un régime harmonique en $\mathrm{e}^{-\mathrm{j} \omega t}$, elles sont données par :

$$
\begin{aligned}
& (\lambda+\mu) \partial \Delta^{\circ} / \partial x+\mu \nabla^{2} \hat{u}=-\rho_{v} \omega^{2} \hat{u} \\
& (\lambda+\mu) 1 / z \partial \Delta^{\circ} / \partial p+\mu \nabla^{2} \hat{v}=-\rho_{y} \omega^{2} \hat{v} \\
& (\lambda+\mu) \partial \Delta^{\circ} / \partial z+\mu \nabla^{2} \hat{\forall}=-\rho_{v} \omega^{2} \hat{\psi}
\end{aligned}
$$


$(\hat{u}, \hat{y}, \hat{w})$ sont les composantes du déplacement d'un point appartenant au matériau souple, respectivement suivant $\vec{x}, \vec{\phi}, \vec{z} ; \rho_{Y}$ est sa masse volumique, $\lambda$ et $\mu$ sont les coefficients de LAME et pour un matériau isotrope sont exprimés à partir du module d'youNG $E_{v}$ et du coefficient de POISSON $v_{y}$ suivant les relations classiques:

$$
\begin{aligned}
& \lambda=E_{v} v_{v}\left(1-j \eta_{\psi}\right) /\left(1+v_{v}\right)\left(1-2 v_{v}\right) \\
& \mu=E_{v}\left(1-j \eta_{\eta}\right) / 2\left(1+v_{v}\right)
\end{aligned}
$$

$\eta_{Y}$ est le facteur de pertes dans la couche visco-élastique, $\Delta^{\circ}$ la dilatation cubique donnée en coordonnées culindriques par:

$$
\Delta^{*}=1 / z \partial(z \hat{W}) / \partial z+1 / z \partial \hat{y} / \partial \varphi+\partial \hat{u} / \partial x
$$

$\nabla^{2}$ est l'opérateur Laplacien que lon prendra en coordonnées cylindriques:

$$
\nabla^{2}=\partial^{2} / \partial z^{2}+1 / z \partial / \partial z+1 / z^{2} \partial^{2} / \partial \psi^{2}+\partial^{2} / \partial x^{2}
$$

\subsection{Déplacements dans la couche viscoélastique}

Introduisons la différence $\varepsilon$, entre le rayon externe de la coque et la cote radiale d'un point du matériau de masquage $: \varepsilon=z-z_{0}$; où $z_{0}$ représente le rayon externe de la coque $(a+$ h/2). Dans le cas où l'épaisseur de la couche est faible, $\varepsilon$ restera petit et les développements asymptotiques des déplacements $\hat{u}, \hat{\forall}, \hat{\psi}$ suivant les puissances de $\hat{\varepsilon}$ pourront être tronqués sans introduire de grosses erreurs. Tronqués à l'ordre 2, les déplacements sont donnés par :

$$
\begin{aligned}
& \hat{u}=\sum_{\alpha=0}^{1} \sum_{n=0}^{\infty} \sum_{m=1}^{\infty}\left(u^{(0)} \alpha n m+\varepsilon u^{(1)} \alpha n m+\varepsilon^{2} / 2 u^{(2)} \alpha n m\right) \\
& \cos m \pi x / L \sin (n \varphi+\alpha \pi / 2) \\
& \left.\hat{v}=\sum_{\alpha=0}^{1} \sum_{n=0}^{\infty} \sum_{m=1}^{\infty} \gamma^{(0)} \alpha \mathrm{nm}+\varepsilon v^{(1)}{ }_{\alpha n m}+\varepsilon^{2} / 2 v^{(2)} \alpha \mathrm{nm}\right) \\
& \sin m \pi x / L \cos (n \varphi+\alpha \pi / 2) \\
& \begin{array}{r}
\hat{w}=\sum_{\alpha=0}^{1} \sum_{n=0}^{\infty} \sum_{m=1}^{\infty}\left(w_{\alpha n m}^{(0)}+\varepsilon w_{\alpha n m}^{(1)}+\varepsilon^{2} / 2 w_{\alpha n m}(2)\right. \\
\quad \sin m \pi x / L \sin (n \varphi+\alpha \pi / 2)
\end{array}
\end{aligned}
$$

Les termes $U^{(s)} \mathrm{anm}^{\prime} \mathrm{W}^{(s)} \mathrm{anm}$ et $\mathrm{w}^{(s)} \mathrm{anm}$ sont assimilables aux dérivées partielles d'ordres $s$ suiwant $\vec{z}$. Exprimés ainsi, c'est-à-dire à partir des modes de coque nue (de caractère $\alpha$, d'ordre circonférentiel $n$, longitudinal $m$ ), ces développements facilitent le raccord avec les déplacements de la coque supportant le matériau, mais surtout autorisent un calcul de rayonnement dans le principe inchangé, ce qui permettra la réutilisation des impédances de rayonnement de la coque nue. 
3.3. Equations modales du mouvement de la couche visco-élastique à l'ordre 0 .

En introduisant (21) dans (17), on obtient (22), (23) et (24), les équations du mouvement qui sont vérifiées à l'ordre 0 (ordre 1 incomplet):

suivant $\vec{x}$ :

$$
\begin{aligned}
& -(\lambda+2 \mu)(m \pi / L)^{2} v_{\alpha n m}^{(0)}+(\lambda+\mu) m \pi / L\left[W^{(1)}{ }_{\alpha n m}+1 / z_{0}\left({ }^{(0)}(0){ }_{\alpha n m}\right.\right.
\end{aligned}
$$

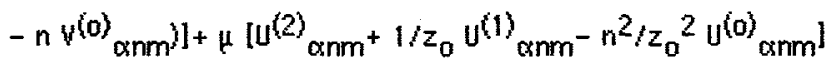

$$
\begin{aligned}
& +p_{v} \omega^{2} u_{\alpha n m}^{(0)}=0
\end{aligned}
$$

suivant $\vec{\phi}$ :

$$
\begin{aligned}
& -(\lambda+2 \mu) n^{2} / z_{0}{ }^{2} v_{\alpha n m}^{(0)}+(h+\mu)\left(n / z_{0}^{2} w_{\alpha n m}^{(0)}+n / z_{0}\left(w_{\alpha n m}^{(1)}{ }_{\alpha n}\right.\right. \\
& \left.\left.-m \pi / L v_{\alpha r m}^{(0)}\right)\right)+\mu\left(\psi_{\alpha n m}^{(2)}+1 / z_{0} v_{\alpha n m}^{(1)}{ }_{\alpha n m}\right. \\
& \left.-(m \pi / L)^{2} v_{\alpha n m}^{(0)}\right)+\rho_{v} \omega^{2} v_{\alpha n m}^{(0)}=0
\end{aligned}
$$

suivant $\vec{z}$ :

$$
\begin{aligned}
& (\lambda+2 \mu)\left(1 / z_{0} w^{(1)}{ }_{\alpha n m}+w(2) w_{\alpha n m}\right)+(\lambda+\mu)\left(-1 / z_{0}^{2} w_{\alpha n m}^{(a)}\right.
\end{aligned}
$$

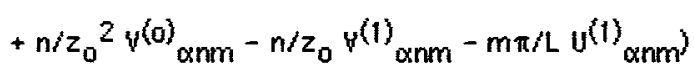

$$
\begin{aligned}
& +\mu\left(-n^{2} / z_{0}^{2} w(0) \alpha n m\right. \\
& \left.-(m \pi / L)^{2} w_{\alpha n m^{(0)}}\right)+p_{\psi}\left(\omega^{2} w_{\alpha n m}^{(0)}=0\right.
\end{aligned}
$$

3.4. Continuité des contraintes à l'interface matériau de masquage-fluide extérieur.

La résolution de notre problème passe par le raccordement des contraintes à l'interface matériau de masquage - fluide extérieur ; soit en $\varepsilon=$ e, où e représente l'épaisseur de la couche. L'état de contrainte sur une facette de normale $\vec{z}$ s'exprime par:

$$
\begin{aligned}
& \sigma_{z z}=(\hat{\lambda}+2 \mu) \partial \hat{w} / \partial z+\lambda(1 / z \partial \hat{y} / \partial \psi+\hat{w} / z+\partial \hat{u} / \partial x) \\
& \sigma_{z x}=\mu(\partial \hat{w} / \partial x+\partial \hat{u} / \partial z) \\
& \sigma_{z y}=\mu(\partial \hat{y} / \partial z-\hat{y} / z+1 / z \partial \hat{\psi} / \partial \varphi)
\end{aligned}
$$

En $\varepsilon=e$, les contraintes prennent la forme suivante lor sque l'on utilise les développements (21) et que l'on raccorde avec la pression acoustique extérieure:

suivant $\vec{x}$ :

$$
\left.m \pi / L(\omega)(0){ }_{\alpha n m}+e w^{(1)}{ }_{\alpha n m}\right)+U^{(1)}{ }_{\alpha n m}+e U_{\alpha n m}^{(2)}=0
$$


suivant $\vec{p}$ :

$$
\begin{aligned}
& v_{\alpha(1)}{ }_{\alpha m}+e w_{\alpha n m}^{(2)}-\left(1 / z_{0}-e / z_{0}{ }^{2}\right) w^{(0)} \alpha n m-e / z_{0} v_{\alpha n m}^{(1)} \\
& +n\left(1 / z_{0}-e / z_{0}{ }^{2}\right) w_{\alpha n m}^{(0)}+e n / z_{0} w_{\alpha n m}^{(1)}=0
\end{aligned}
$$

suivant $\vec{z}$ :

$$
\begin{aligned}
& (\lambda+2 \mu)\left(\omega^{(1)} \mathrm{cnm}+e w^{(2)} \mathrm{anm}\right)+\lambda\left(-n\left(1 / z_{0}-e / z_{0}{ }^{2} w^{(0)} \mathrm{anm}\right.\right.
\end{aligned}
$$

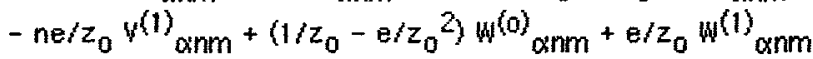

$$
\begin{aligned}
& \left.-m \pi / L U^{(0)}{ }_{a n m}+e U_{\alpha n m}^{(1)}\right) \\
& =j \omega \varepsilon_{n} / \pi L\left(z_{0}+e\right) \sum_{r=1}^{\infty} z_{n m r}\left(w(0){ }_{\alpha n r}+e w(1)_{\alpha n r}+e^{2} / 2 w(2){ }_{\alpha n r}\right)
\end{aligned}
$$

Au deuxième membre de (28) apparaìt l'impédance de rayonnement madale, qui résulte du raccordement implicite des déplacements radiaux à l'interface fluide - couche de masquage.

3.5. Continuité des déplacements à l'interface matériau de masquage - caque et équations modales du mouvement de la coque recouverte de matériau

\section{a) Conditions aux limites en déplacement à l'interface $\hat{z}=0$}

On doit satisfaire la continuité des déplacements entre la face externe de la coque (de rayon $z_{0}$ ) et la face interne du matériau de masquage, ce qui permet d'établir pour chaque ordre $\alpha, n, m$ les relations:

$$
\begin{aligned}
& u^{(0)}{ }_{\mathrm{rrm}}=\sum_{j=1}^{3} a_{n m j}^{\alpha}\left(D_{n m j}-m \pi h / 2 L\right) \\
& v_{a n m}^{(0)}=\sum^{3} a^{\alpha}{ }_{n m j}\left(E_{n m j}-n h / 2 a\right) \\
& \mathrm{j}=1 \\
& w_{\mathrm{wrm}}^{(0)}=\sum^{3} a^{\alpha}{ }_{\mathrm{nm} j} \\
& j=1
\end{aligned}
$$

b) Equations modales du mouvement de la coque raidie recouvertes d'un materiau de masquage

Elles prennent la forme suivante:

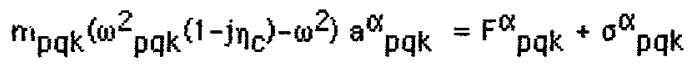

où $\sigma_{p q k}^{\alpha}$ définit la force généralisée liée au champ de contrainte appliquée à la coque par le matériau de masquage qui yaut: 


$$
\begin{aligned}
& { }_{0}^{\alpha}{ }_{p q k}=\pi L z_{0} / \varepsilon_{p}\left[(\lambda+2 \mu) w^{(1)}{ }_{\alpha p q}+\lambda\left(-p / z_{0} v^{(0)}{ }_{\alpha p q}+w^{(0)}{ }_{\alpha p q} / z_{0}\right.\right. \\
& \left.-q \pi / L u^{(0)}{ }_{\alpha p q}\right)+\mu\left(0_{\rho q k}-q \pi / L h / 2\right)\left(q \pi / L w_{\alpha p q}(0){ }_{\alpha p q}^{(1)}{ }_{\alpha p q}\right. \\
& \left.+\mu\left(E_{p q k}-p / a n / 2\right)\left(\psi^{(1)} \alpha p q-\psi_{\alpha p q}(0) z_{0}+p / z_{0}(0){ }_{\alpha p q}\right)\right]
\end{aligned}
$$

La résolution du système linéaire formé par les équations $(22,23,24,26,27,28,30)$ dans lesquelles les substitutions (29) ont été effectuées permet de calculer les amplitudes modales puis de remonter aux grandeurs caractéristiques d'un problème de rayonnement, soit la puissance acoustique emise, et la vitesse quadratique radiale de la coque et de la couche de masquage.

\section{RESULTATS NUMERIQUES ET COMPARAISON DES DEUX MODELES}

Nous présentons en figures 2 et 3 des résultats en fonction de la fréquence concernant la puissance acoustique rayonnée $L_{W}$ et la vitesse quadratique radiale $L_{Y}$ d'une coque en acier, Je longueur $1.2 \mathrm{~m}$, d'épaisseur $3 \mathrm{~mm}$, de rayon $0.4 \mathrm{~m}$, recouverte d'un matériau de masquage d'impédance localisée fixée à $Z=510^{8} \mathrm{~N} / \mathrm{m}^{3}$, son facteur de perte valant $10^{-2}$, et non recouverte $\left(Z\right.$ infini). La coque est immergée $\left(C=1500 \mathrm{~m} / \mathrm{s}, \rho_{0}=1000 \mathrm{~kg} / \mathrm{m}^{3}\right)$ et est êtudiée jusqu'à sa fréquence d'anneau (2000 Hz).

On constate que d'une part, cette valeur d'impédance réduit les niveaux de puissance rayonnée presque partout, à part en basse iréquence $(50-150 \mathrm{~Hz})$ et vers $850 \mathrm{~Hz}$. La vitesse de coque est fortement perturbée par lapplication du matériau et on observe une forte diminution du niveau vibratoire vers $600-700 \mathrm{~Hz}$, qui s'apparente à un phénomène d'antirésonance. Inversement au-dessus de $800 \mathrm{~Hz}$, la vitesse de coque est plus forte que celle de la coque nue, signe que le materiau isole la coque du fluide extérieur (diminution de la charge fluide et des dissipations par rayonnement).

Il apparait difficile de comparer les deux méthodes de calcul tellement les équations qui les régissent sont différentes. Néanmoins, définissons une impédance équivalente au matériau de masquage modèlisé par l'élasticité tridimensionnelle. L'impédance localisée, en Newton par $\mathrm{m}^{3}$, définissait, somme toute, le rapport du module d'Young du matériau divisé par son épaisseur, ce qui revient à dire que seul le rapport de ces deux grandeurs était connu. Avec l'approche tridimensionneile, définissons son impédance équivalente par le rapport:

$$
Z_{\overline{E q}}=(\lambda+2 \mu) / \mathrm{e}
$$

ce qui revient à négliger le couplage avec les directions transwersales $(\vec{x}, \vec{p})$ dans l'expression de la contrainte radiale $\sigma_{z z}$ :

Nous présentons en figure (4), un résultat établi par l'approche tridimensionnelle pour la même coque, munie d'une couche visco-élastique de coefficent de poIsson nul, ce qui nous place dans la configuration la plus proche de la modèlisation par impédance localisée. On observe les mêmes tendances d'évolution de la puissance en fonction de la fréquence, bien que des différences sensibles apparaissent. Il nest done pas possible de représenter une couche yisco-élastique, même de coefficient de POISSON nul par une impédance localisée. 


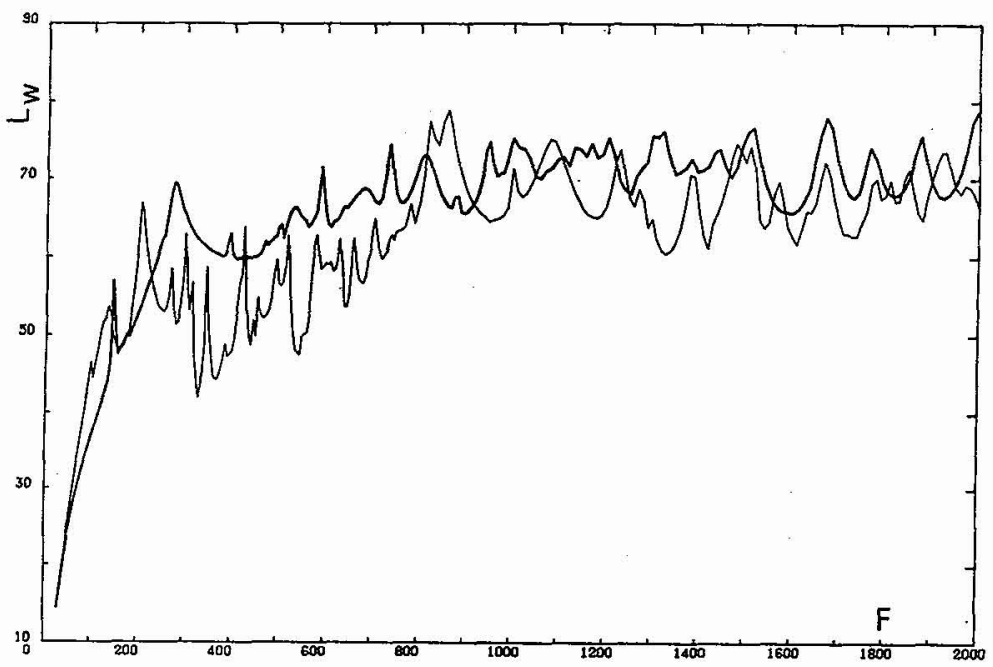

Figure 2 : Puissance rayonnée par la coque dans l'eau —coque nue ; _ coque recouverte de matériau à impédance localisée, $z=510^{8} \mathrm{~N} / \mathrm{m}^{3}, n_{V}=10^{-2}$

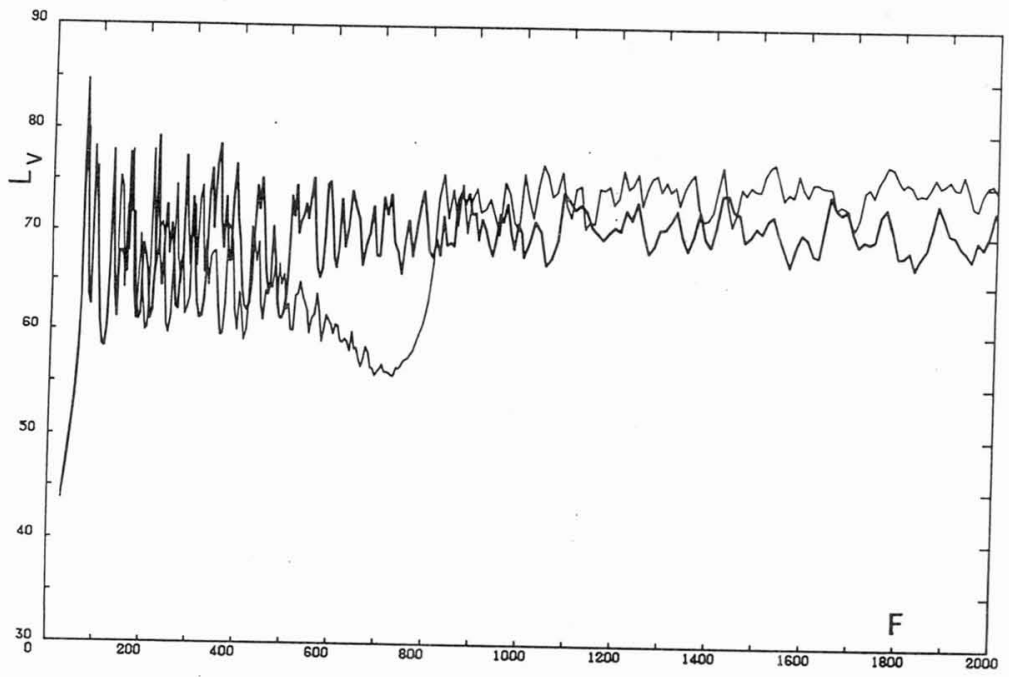

Figure 3 : Vitesse quadratique radiale de coque dans l'eau - coque nue ; - coque recouyerte de matériau à impédance localisée,

$$
Z=510^{8} \mathrm{~N} / \mathrm{m}^{3}, \eta_{Y}=10^{-2}
$$




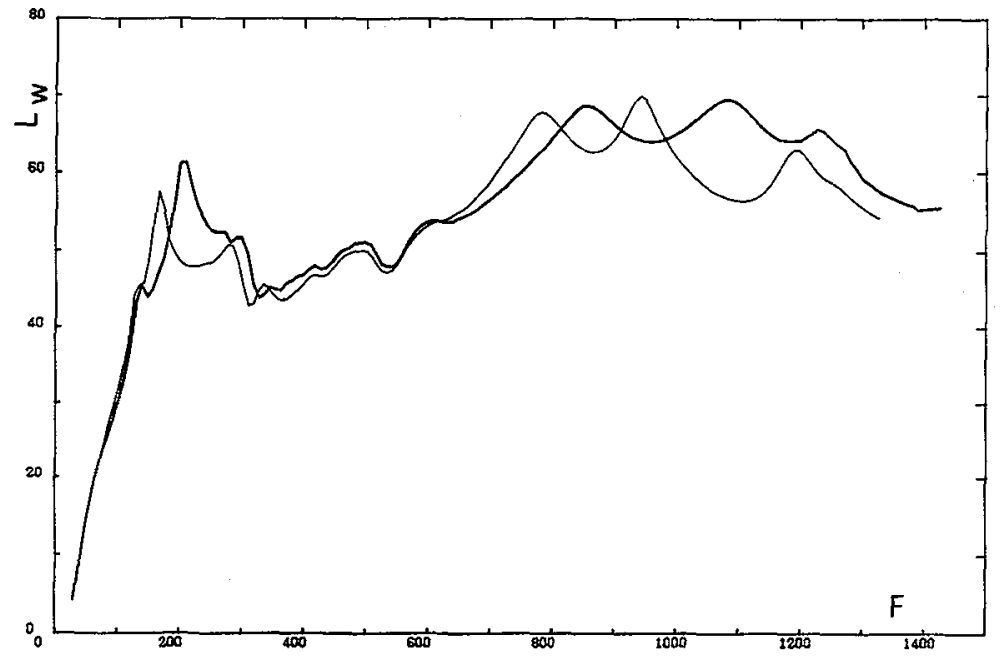

Figure 4 : Puissance rayonnée par la coque recouver te de matériau de masquage ;

- impédance localisée : $Z=510^{8} \mathrm{~N} / \mathrm{m}^{3}, \eta_{Y}=0.1$;

- approche tridimensionnelle $Z_{e q}=510^{8} \mathrm{~N}^{\prime} \mathrm{m}^{3}, \eta_{V}=0.1, v=0, e=1 \mathrm{~cm}$

$$
E_{Y}=510^{6} \mathrm{~N} / \mathrm{m}^{2}, \rho_{Y}=0
$$

\section{CONCLUSION}

Cet article a présenté l'étude du rayonnement acoustique d'une coque recouverte d'un matériau de masquage. La modèlisation théorique du matériau a été effectuée, soit suivant la démarche à réaction localisée, soit par une méthode basée sur le développement asymptotique des équations de l'élasticité tridimensionnelle. Les résultats ont montré que les possiblités de réduction du bruit à la source existaient (dans la mesure ou l'on ne s'imposait aucune contrainte sur la compressibilité du matériaul. Sur un cas particulier, les modèles à impédance localisée et tridimensionnelles ont été comparés, approches qui conduisent à des différences sensibles en puissance acoustique rayonnée.

\section{BIBLIOGRAPHIE}

[1] LAULAGNET B., GUYAOER J.L., J.S.Y., 1989, 131 (3)

[2] LAULAGNET B., Thèse Sciences, 1989, $194 \mathrm{p}$.

[3] LAULAGNET B., GUYADER J.L., A paraître, J.S.V.

[4] LIU Y.N., TUCKER A.J.,NOISE-CON 1988,WEST-LAFAYETTE U.S. A

[5] LAULAGNET B., GUYADER J.L., A paraittre, J. A. S.M.E.

(Acoustics, Yibration, Stress and Reliability in Design)

[6] LOVE A.E.H., New York Doyer Publications, 1944, 643 p

\section{REMERCIEMENTS}

Les auteurs veulent exprimer leur gratitude à la Direction Recherches, Etudes et Techniques qui a supporté ce travail. 